\title{
Resolution threshold for coherent sources using smoothed eigenstructure methods
}

\author{
L. Chang \\ C.-C. Yeh
}

Indexing terms: Signal processing, MUSIC, Mathematical techniques, Array processing, Direction finding

\begin{abstract}
The paper presents a performance analysis of certain eigenstructure based methods (MUSIC type) with suitable forward-only and forward/backward smoothing schemes employed to decorrelated coherent sources. Bias and resolution thresholds are derived for two coherent sources with different phase and unequal power. The effect of phase difference and power ratio on the resolution threshold is discussed. For the case of forward/backward smoothing and two coherent sources with same phase and equal power, the resolution threshold reduces to that obtained by Pillai et al. Detailed computer simulations which confirm the analysis are also presented.
\end{abstract}

\section{Introduction}

High-resolution eigenstructure based techniques [1-6] can estimate the directions of arrival (DOAs) of incoherent and partially coherent sources. For coherent sources, these techniques may be modified by preprocessing smoothing techniques [7-9], which can decorrelate the coherence between sources. The eigenstructure-based techniques can resolve any two closely spaced sources when the ensemble covariance matrix is available. However, in reality, the covariance matrix is estimated from finite array sample data. The deviation between the estimated and the ensemble covariance matrices produces a biased estimation, and these techniques have different performances.

Recently, Kaveh et al. [10] derived the expressions of the bias and resolution threshold, the signal-to-noise ratio (SNR) at which two closely spaced sources can be resolved, of the MUSIC method for two uncorrelated sources. Later Pillai et al. $[11,12]$ presented the bias and resolution threshold of the MUSIC method with a forward/backward smoothing scheme for two coherent sources of equal power and the same phase. Since two coherent sources usually have different phase and power, we derive in this paper the bias and resolution thresholds of the MUSIC method for such two coherent sources. Instead of solving for eigencomponents of the covariance matrix, the derivation involves the sum and product of the two largest eigenvalues. The bias and resolution

Paper 8089F (E5, E15), first received 4th May and in revised form 30th November 1990

The authors are with the Department of Electrical Engineering, National Taiwan University, Taipei, Taiwan, Republic of China thresholds are obtained for both the forward/backward and forward-only smoothing schemes.

\section{Problem formulation and previous results}

Consider a uniform linear array illuminated by two coherent narrow-band sources with complex waveforms $u(t)$ and $|\rho| e^{j \phi} u(t)$ and DOAs $\theta_{1}$ and $\theta_{2}$ with respect to the array broadside, where $|\rho|$ and $\phi$ represent the relative amplitude attenuation and phase delay of the second source with respect to the first source. Without loss of generality, let us assume that $|\rho| \leqslant 1$. To estimate the DOAs of the two coherent sources, the array is grouped into $K$ subarrays, each with $M$ sensors. With the assumption that the signals and noises are stationary, zero mean circular Gaussian independent random processes and, further, the noises are IID (independent and identically distributed) with common variance $\sigma^{2}$, the ensemble forward smoothed covariance matrix of the $K$ subarrays can be written as

$$
\bar{R}_{f}=A\left[\frac{1}{K} \sum_{k=1}^{K} D^{k-1} R_{u}\left(D^{k-1}\right)^{H}\right] A^{H}+\sigma^{2} I
$$

where

$$
\begin{aligned}
A & =\sqrt{ }(M)\left[a\left(\omega_{1}\right), a\left(\omega_{2}\right)\right] \\
D & =\operatorname{diag}\left[e^{-j \omega_{1}}, e^{-j \omega_{2}}\right] \\
R_{u} & =E\left[|u(t)|^{2}\right]\left(\begin{array}{cc}
1 & |\rho| e^{-j \phi} \\
|\rho| e^{j \phi} & |\rho|^{2}
\end{array}\right)
\end{aligned}
$$

In the above equations, $E$ and $H$ denote expectation and hermitian, respectively, and $a\left(\omega_{i}\right)$ is the normalised direction vector associated with $\theta_{i}$,

$$
a\left(\omega_{i}\right)=\frac{1}{\sqrt{ } M}\left[1, e^{-j \omega_{i}}, e^{-j 2 \omega_{i}}, \ldots, e^{-j(M-1) \omega_{i}}\right]^{T}
$$

where

$$
\omega_{i}=\frac{2 \pi d \sin \theta_{i}}{\lambda} \quad i=1,2
$$

$d=$ the space between sensors and $\lambda=$ the wavelength of the signal sources.

It is known that in $\bar{R}_{f}$ the two coherent sources are decorrelated when $K \geqslant 2$. To reduce the number of extra sensors required for smoothing, the ensemble forward/ backward smoothed covariance matrix can be formed as

$$
\begin{aligned}
\bar{R} & =\frac{1}{2}\left\{\bar{R}_{f}+\tilde{I}_{\bar{R}}^{*} \tilde{I}\right\} \\
& =A \bar{R}_{u} A^{H}+\sigma^{2} I
\end{aligned}
$$


where $\tilde{I}$ is the reverse permutation matrix

$$
\tilde{I}=\left[\begin{array}{ccccc}
0 & \cdots & \cdots & 0 & 1 \\
0 & \cdots & 0 & 1 & 0 \\
\vdots & \vdots & & & \vdots \\
0 & 1 & 0 & \cdots & 0 \\
1 & 0 & \cdots & \cdots & 0
\end{array}\right]
$$

and

$$
\begin{aligned}
\bar{R}_{u}=\frac{1}{2 K}[ & \sum_{k=1}^{K} D^{k-1} R_{u}\left(D^{k-1}\right)^{H} \\
& \left.+\sum_{k=1}^{K} D^{-(K+M-k-1)} R_{u}^{*}\left(D^{-(K+M-k-1)}\right)^{H}\right]
\end{aligned}
$$

with '*' representing conjugation. In $\bar{R}$, the two coherent sources are decorrelated with $K \geqslant 1$ except when the phase difference between the two coherent sources is either $0^{\circ}$ or $180^{\circ}$ at the array aperture centre in the case of $K=1$.

The MUSIC method can estimate the DOAs of the two coherent sources using either the forward-only smoothed or the forward/backward smoothed covariance matrices. With the ensemble covariance matrices $\bar{R}_{f}$ and $\widetilde{R}$ described as above, the MUSIC method can resolve any two closely spaced coherent sources. Practically, the covariance matrix is estimated from array sample data. The difference between the estimated covariance matrix and the ensemble covariance matrix results in a biased estimation of DOAs. Recently, Pillai et al. [12] derived the general expressions for the mean and variance of the null spectrum of the MUSIC method. For two coherent sources, the mean and variance are given by

$$
\begin{aligned}
& E[\hat{Q}(\omega)] \\
& =Q(\omega)+\frac{1}{N} \sum_{i=1}^{2}\left[\sum_{\substack{k=1 \\
k \neq i}}^{M} \frac{\Gamma_{i i k k}}{\left(\lambda_{i}-\lambda_{k}\right)^{2}}\left|\beta_{i}^{H} a(\omega)\right|^{2}\right. \\
& \left.\quad-\sum_{\substack{k=1 \\
k \neq i}}^{M} \sum_{\substack{l=1 \\
l \neq i}}^{M} \frac{\Gamma_{i i k l}}{\left(\lambda_{i}-\lambda_{k}\right)\left(\lambda_{i}-\lambda_{l}\right)} a^{H}(\omega) \beta_{k} \beta_{l}^{H} a(\omega)\right] \\
& \quad+O\left(\frac{1}{N^{2}}\right)
\end{aligned}
$$

and

$$
\begin{aligned}
\operatorname{Var}[\hat{Q}(\omega)] & \frac{2}{N} \sum_{i=1}^{2} \sum_{j=1}^{2} \sum_{\substack{k=1 \\
k \neq i}}^{M} \sum_{\substack{l=1 \\
l \neq j}}^{M} a^{H} \beta_{k} \beta_{i}^{H} a \\
& \times \frac{\operatorname{Re}\left[\Gamma_{k l j i} a^{H}(\omega) \beta_{j} \beta_{l}^{H} a(\omega)+\Gamma_{k j l i} a^{H}(\omega) \beta_{l} \beta_{j}^{H} a(\omega)\right]}{\left(\lambda_{i}-\lambda_{k}\right)\left(\lambda_{j}-\lambda_{l}\right)} \\
& +O\left(\frac{1}{N^{2}}\right)
\end{aligned}
$$

In eqns. 10 and $11, N$ denotes the number of snapshots, $\lambda_{i}$ and $\beta_{i}, i=1,2, \ldots, M$, are the eigenvalues in descending order and associated eigenvectors, respectively, of the ensemble smoothed covariance matrix, $\operatorname{Re}(\cdot)$ stands for the real part of $(\cdot)$, and $Q(\omega)$ is the null spectrum computed from the ensemble smoothed covariance matrix, i.e.

$$
\begin{aligned}
Q(\omega) & =\sum_{i=3}^{M}\left|\beta_{i}^{H} a(\omega)\right|^{2} \\
& =1-\sum_{i=1}^{2}\left|\beta_{i}^{H} a(\omega)\right|^{2}
\end{aligned}
$$

Here, $\Gamma_{i k l j}$ is given by

$$
\Gamma_{i k l j}=\left\{\begin{array}{ccc}
\frac{1}{4 K^{2}} \sum_{p=1}^{K} \sum_{q=1}^{K} \beta_{i}^{H} R_{p q}^{f} \beta_{k} \beta_{l}^{H} R_{q p}^{f} \beta_{j} & \\
& +\beta_{i}^{H} R_{p q}^{b} \beta_{k} \beta_{l}^{H} R_{q p}^{b} \beta_{j} & \\
+\beta_{i}^{H} R_{p q 0}^{f} \gamma_{l} \gamma_{j}^{H} R_{p 0 q}^{b} \beta_{k} & \text { for forward/ } \\
+\beta_{i}^{H} R_{p q o}^{b} \gamma_{l} \gamma_{j}^{H} R_{p 0 q}^{f} \beta_{k} & \text { sackward } \\
\frac{1}{K^{2}} \sum_{p=1}^{K} \sum_{q=1}^{K} \beta_{i}^{H} R_{p q}^{f} & \text { smoothing } \\
\times \beta_{k} \beta_{l}^{H} R_{q p}^{f} \beta_{j} & \text { or forward- }
\end{array}\right.
$$

In eqn. $13, p_{0}=K-p+1, q_{0}=K-q+1$ and $\gamma_{j}$ is the backward version of the vector $\beta_{j}^{*}$. Further,

$$
\begin{aligned}
& R_{p q}^{f}=E\left[X_{p}^{f}\left(X_{q}^{f}\right)^{H}\right] \\
& R_{p q}^{b}=E\left[X_{p}^{b}\left(X_{q}^{b}\right)^{H}\right]
\end{aligned}
$$

where $X_{i}^{f}$ (or $X_{i}^{b}$ ) is the output vector of the $i$ th forward (or backward) subarray. For the forward/backward smoothing with $K=1$, eqn. 10 reduces to

$$
\begin{aligned}
E[\hat{Q}(\omega)]= & Q(\omega)+\frac{1}{2 N} \sum_{i=1}^{2} \frac{\lambda_{i} \sigma^{2}}{\left(\lambda_{i}-\sigma^{2}\right)^{2}} \\
& \times\left[(M-2)\left|\beta_{i}^{H} a(\omega)\right|^{2}-Q(\omega)\right] \\
& +O\left(\frac{1}{N^{2}}\right)
\end{aligned}
$$

Since $\operatorname{Var}\left[\hat{Q}\left(\omega_{k}\right)\right]$ is much less than $E\left[\hat{Q}\left(\omega_{k}\right)\right]$ for $k=1,2$ [12], the resolution threshold for two closely spaced sources is defined as the smallest SNR for which the following inequality is satisfied [10]

$$
E\left[\hat{Q}\left(\omega_{m}\right)\right] \geqslant E\left[\hat{Q}\left(\omega_{i}\right)\right] \quad i=1,2
$$

where $\omega_{m}=\left(\omega_{1}+\omega_{2}\right) / 2$. From eqns. 15 and 16 , Pillai et al. have derived the resolution threshold $\tilde{\xi}_{T}$ for two coherent sources of equal power and same phase, to be [12]

$$
\tilde{\xi}_{T} \approx\left(\frac{1}{3} \Delta^{-2}-\frac{1}{20}-\frac{1}{16} \Delta^{2}\right) \xi_{T}
$$

where $\xi_{T}[10]$ is the normalised SNR (resolution threshold) required to resolve two equipowered uncorrelated sources separated by the normalised 'angle' $\Delta^{2}=$ $\left(\boldsymbol{M}^{2} \omega_{d}^{2}\right) / 3.2 \omega_{d}=\left(\omega_{2}-\omega_{1}\right)$ represents the actual separation.

In reality, two coherent sources usually have different phase and power. We analyse in this paper the performance of the MUSIC method with forward/backward and forward-only smoothing schemes for such coherent sources. The number of subarrays $K$ is chosen to be one for forward/backward smoothing and two for forwardonly smoothing (i.e. the minimum required).

\section{Performance of MUSIC with forward/backward smoothing}

The expressions of the bias and resolution threshold for the MUSIC scheme with forward/backward smoothing and $K=1$ for two coherent sources with different phase and power are derived below. $E\left[\hat{Q}\left(\omega_{i}\right)\right], i=1,2$, is first evaluated by using eqn. 15 which is the general expression for the MUSIC scheme with forward/backward smoothing and $K=1$ in the case of two coherent 
sources. Eqn. 15 involves the eigenvalues and eigenvectors associated with the signal subspace of the smoothed ensemble covariance matrix $\bar{R}$. For the case considered here, the expressions of the eigenvalues and eigenvectors are too complex to be used for evaluating the bias. In the analysis shown below, that problem is circumvented by using the sum and product of the two largest eigenvalues.

To evaluate $E\left[\hat{Q}\left(\omega_{i}\right)\right],\left|\beta_{j}^{H} a\left(\omega_{i}\right)\right|, i, j=1,2$, is first examined. Let $\mu_{i}$ be the eigenvalue of the noise free ensemble covariance matrix $A \bar{R}_{u} A^{H} . \mu_{i}$ is related to $\lambda_{i}$, the eigenvalue of $\bar{R}$, by

$$
\mu_{i}=\lambda_{i}-\sigma^{2}
$$

Using eqns. 2, 4 and $9, A \bar{R}_{u} A^{H}$ can be written as

$$
\begin{aligned}
A \bar{R}_{u} A^{H}= & M E\left[|u(t)|^{2}\right]\left[a\left(\omega_{1}\right) a\left(\omega_{2}\right)\right] \\
& \times\left(\begin{array}{cc}
1 & |\rho| \rho_{t} \\
|\rho| \rho_{t}^{*} & |\rho|^{2}
\end{array}\right)\left[a\left(\omega_{1}\right) a\left(\omega_{2}\right)\right]^{H} \\
= & M E\left[|u(t)|^{2}\right]\left[\begin{array}{ll}
\beta_{1} & \beta_{2}
\end{array}\right] \\
& \times\left(\begin{array}{cc}
\mu_{1}^{\prime} & 0 \\
0 & \mu_{2}^{\prime}
\end{array}\right)\left[\begin{array}{ll}
\beta_{1} & \left.\beta_{2}\right]^{H}
\end{array}\right.
\end{aligned}
$$

In eqn. $19, \rho_{2}$ is the effective correlation coefficient of the two coherent sources for forward/backward smoothing and $K=1$

$$
\begin{aligned}
& \rho_{1}=e^{-j(M-1) \omega_{d}} \cos \left[(M-1) \omega_{d}-\phi\right] \\
& E\left[\hat{Q}\left(\omega_{m}\right)\right]=Q\left(\omega_{m}\right)+\frac{M-2}{2 N}\left\{\frac{1}{\zeta}|\rho|^{2} \frac{\left(\mu_{1}^{\prime}+\mu_{2}^{\prime}\right)-S i^{2}[M}{\zeta^{2}}|\rho|^{4} \frac{\left(\mu_{1}^{\prime 2}+\mu_{1}^{\prime} \mu_{2}^{\prime}+\mu_{2}^{\prime 2}\right)-S i^{2}\left[M,\left(\omega_{d} / 2\right)\right]}{\mu_{1}^{\prime 2} \mu_{2}^{\prime 2}}\right. \\
& +\frac{1}{\text { and } \mu_{i}^{\prime} \text { is defined as }} \\
& \mu_{i}^{\prime}=\frac{\mu_{i}}{M E\left[|u(t)|^{2}\right]} \\
& \begin{array}{l}
\text { Premultiplying and postmultiplying eqn. } 19 \text { by } a^{H}\left(\omega_{i}\right) \text { and } \\
\text { at } \left.\omega_{i}\right), \text { respectively, for } i=1,2, \text { we get the following equa- } \\
\text { tions } \quad=\mu_{1}^{\prime}\left|\beta_{1}^{H} a\left(\omega_{1}\right)\right|^{2}+\mu_{2}^{\prime}\left|\beta_{2}^{H} a\left(\omega_{1}\right)\right|^{2}
\end{array} \\
& 1+|\rho|^{2}\left|\rho_{\mathrm{s}}\right|^{2}+|\rho| \rho_{t} \rho_{\mathrm{s}}+|\rho| \rho_{t}^{*} \rho_{s}^{*}
\end{aligned}
$$$$
E\left[\hat{Q}\left(\omega_{m}\right)\right]=Q\left(\omega_{m}\right)+\frac{M-2}{2 N}\left\{\frac{1}{\zeta}|\rho|^{2} \frac{\left(\mu_{1}^{\prime}+\mu_{2}^{\prime}\right)-S i^{2}\left[M,\left(\omega_{d} / 2\right)\right] \rho_{a}-Q\left(\omega_{m}\right) \mu_{1}^{\prime}}{\mu_{1}^{\prime} \mu_{2}^{\prime}}\right.
$$$$
\left.+\frac{1}{\zeta^{2}}|\rho|^{4} \frac{\left(\mu_{1}^{\prime 2}+\mu_{1}^{\prime} \mu_{2}^{\prime}+\mu_{2}^{\prime 2}\right)-S i^{2}\left[M,\left(\omega_{d} / 2\right)\right] \rho_{a}\left(\mu_{1}^{\prime}+\mu_{2}^{\prime}\right)-Q\left(\omega_{m}\right) \mu_{1}^{\prime 2}}{\mu_{1}^{\prime 2} \mu_{2}^{\prime 2}}\right\}
$$

and

$$
\begin{aligned}
|\rho|^{2}+\left|\rho_{s}\right|^{2}+|\rho| \rho_{t} \rho_{s}+|\rho| \rho_{t}^{*} \rho_{s}^{*} \\
\quad=\mu_{1}^{\prime}\left|\beta_{1}^{H} a\left(\omega_{2}\right)\right|^{2}+\mu_{2}^{\prime}\left|\beta_{2}^{H} a\left(\omega_{2}\right)\right|^{2}
\end{aligned}
$$

In eqns. 22 and $23, \rho_{s}$ is defined as

$$
\rho_{s}=a^{H}\left(\omega_{2}\right) a\left(\omega_{1}\right)=e^{j(M-1) \omega_{d}} \operatorname{Si}\left(M, \omega_{d}\right)
$$

where

$$
\operatorname{Si}(x, y) \triangleq \frac{\sin (x y)}{x \sin y}
$$

Since $\beta_{1}$ and $\beta_{2}$ span the signal subspace, we have

$$
\left|\beta_{1}^{H} a\left(\omega_{i}\right)\right|^{2}+\left|\beta_{2}^{H} a\left(\omega_{i}\right)\right|^{2}=1 \text { for } i=1,2
$$

Solving eqns. 22,23 and 26 , we get

$$
\begin{aligned}
& \left|\beta_{j}^{H} a\left(\omega_{i}\right)\right|^{2}=(-1)^{j} \frac{|\rho|^{2(2-i)}\left(1-\left|\rho_{s}\right|^{2}\right)-\mu_{j}^{\prime}}{\mu_{1}^{\prime}-\mu_{2}^{\prime}} \\
& \qquad \text { for } i, j=1,2
\end{aligned}
$$

Substituting eqn. 27 into eqn. 15, the mean of the null spectrum in the directions of $\omega_{1}$ and $\omega_{2}$ can be obtained within the first-order approximation, as

$$
\begin{aligned}
E\left[\hat{Q}\left(\omega_{i}\right)\right]= & \frac{M-2}{2 N}\left\{-\frac{|\rho|^{4}}{\zeta^{2} \mu_{1}^{\prime} \mu_{2}^{\prime}}+\frac{|\rho|^{2(3-i)}\left(1-\left|\rho_{s}\right|^{2}\right)}{\zeta \mu_{1}^{\prime} \mu_{2}^{\prime}}\right. \\
& \left.\times\left[1+\frac{|\rho|^{2}\left(\mu_{1}^{\prime}+\mu_{2}^{\prime}\right)}{\zeta \mu_{1}^{\prime} \mu_{2}^{\prime}}\right]\right\} \text { for } i=1,2
\end{aligned}
$$

where $\zeta$ is defined as the array output SNR of the weaker source,

$$
\zeta \triangleq \frac{M E\left[|u(t)|^{2}\right]|\rho|^{2}}{\sigma^{2}}
$$

Similarly, to find the bias in the direction of $\omega_{m}$, the factor $\left|\beta_{i}^{H} a\left(\omega_{m}\right)\right|^{2}$ can be evaluated by premultiplying and postmultiplying eqn. 19 by $a^{H}\left(\omega_{m}\right)$ and $a\left(\omega_{m}\right)$, respectively, and this results in

$$
\begin{aligned}
\left|\beta_{1}^{H} a\left(\omega_{m}\right)\right|^{2} & \simeq \frac{-\mu_{2}^{\prime}+S i^{2}\left[M,\left(\omega_{d} / 2\right)\right] \rho_{a}}{\mu_{1}^{\prime}-\mu_{2}^{\prime}} \\
\left|\beta_{2}^{H} a\left(\omega_{m}\right)\right|^{2} & \simeq \frac{\mu_{1}^{\prime}-S i^{2}\left[M,\left(\omega_{d} / 2\right)\right] \rho_{a}}{\mu_{1}^{\prime}-\mu_{2}^{\prime}}-Q\left(\omega_{m}\right)
\end{aligned}
$$

where $\rho_{a}$ is given by

$$
\rho_{a}=1+|\rho|^{2}+|\rho| \rho_{t} e^{j(M-1) \omega_{d}}+|\rho| \rho_{t}^{*} e^{-j(M-1) \omega_{d}}
$$

From eqns. 30 and 31 , we may obtain the $E\left[\hat{Q}\left(\omega_{m}\right)\right]$ with the first-order approximation
Since $Q\left(\omega_{m}\right)$ is much less than unity for two closely spaced sources and $\mu_{2}^{\prime}$ is smaller than $\mu_{1}^{\prime}, Q\left(\omega_{m}\right) \mu_{1}^{\prime}$ and $Q\left(\omega_{m}\right)\left(\mu_{1}^{\prime}\right)^{2}$ in eqn. 33 can be approximated by $Q\left(\omega_{m}\right)\left(\mu_{1}^{\prime}+\mu_{2}^{\prime}\right)$ and $Q\left(\omega_{m}\right)\left(\mu_{1}^{\prime}+\mu_{2}^{\prime}\right)^{2}$, respectively. Therefore, from eqns. 28 and $33, E[\hat{Q}(\omega)], \omega=\omega_{1}, \omega_{2}$ and $\omega_{m}$, is only related to the sum and product of $\mu_{i}^{\prime}, i=1,2$. Since $\mu_{1}$ and $\mu_{2}$ are eigenvalues of $A \bar{R}_{u} A^{H}$, the following equation holds

$$
\mu_{i}^{2}-\operatorname{Tr}\left(A \bar{R}_{u} A^{H}\right) \mu_{i}+\left|\bar{R}_{u} A^{H} A\right|=0
$$

where $\operatorname{Tr}(\cdot)$ denotes the trace of $(\cdot)$. From eqns. 21 and 34 , the sum and product of $\mu_{1}^{\prime}$ and $\mu_{2}^{\prime}$ are given by

$$
\begin{aligned}
\mu_{1}^{\prime}+\mu_{2}^{\prime} & =\frac{\operatorname{Tr}\left(A \bar{R}_{u} A^{H}\right)}{M E\left[|u(t)|^{2}\right]} \\
& =1+|\rho|^{2}+|\rho|\left(\rho_{s} \rho_{t}+\rho_{s}^{*} \rho_{t}^{*}\right) \\
\mu_{1}^{\prime} \mu_{2}^{\prime}= & \frac{\left|\bar{R}_{u} A^{H} A\right|}{\left\{M E\left[|u(t)|^{2}\right]\right\}^{2}} \\
= & |\rho|^{2}\left(1-\left|\rho_{s}\right|^{2}\right)\left(1-\left|\rho_{t}\right|^{2}\right)
\end{aligned}
$$

One can see from eqn. 28 that $E\left[\hat{Q}\left(\omega_{2}\right)\right] \geqslant E\left[\hat{Q}\left(\omega_{1}\right)\right]$ for $|\rho|^{2} \leqslant 1$. Therefore, the resolution threshold is determined by the weaker source,

$$
E\left[\hat{Q}\left(\omega_{m}\right)\right] \geqslant E\left[\hat{Q}\left(\omega_{2}\right)\right]
$$

which is the same as the conclusion drawn by Lee [13] for two uncorrelated sources. Substituting eqns. 28 and 33 into eqn. 37 and using $Q\left(\omega_{m}\right) \simeq \frac{1}{80} \Delta^{4}[10,13]$, we may 
get the resolution threshold,

$$
\zeta=\frac{20(M-2)}{N \Delta^{4}} A\left\{1+\sqrt{\left.\left[1+\frac{N \Delta^{4}}{10(M-2)} B\right]\right\}}\right.
$$

where $A$ and $B$ are given by

$$
A \simeq \frac{1-\left|\rho_{s}\right|^{2}+S i^{2}\left[M,\left(\omega_{d} / 2\right)\right] \rho_{a}+\left(Q\left(\omega_{m}\right)-1\right)\left(\mu_{1}^{\prime}+\mu_{2}^{\prime}\right)}{\left(1-\left|\rho_{s}\right|^{2}\right)\left(1-\left|\rho_{t}\right|^{2}\right)}
$$

and

$$
B \simeq \frac{\mu_{1}^{\prime}+\mu_{2}^{\prime}}{1-\left|\rho_{s}\right|^{2}+S i^{2}\left[M,\left(\omega_{d} / 2\right)\right] \rho_{a}+\left(Q\left(\omega_{m}\right)-1\right)\left(\mu_{1}^{\prime}+\mu_{2}^{\prime}\right)}
$$

For two closely spaced sources, it can be shown that the resolution threshold may be approximated as follows.

(a) For $|\sin \phi| \ll \Delta$ :

$$
\begin{aligned}
\zeta \simeq & \frac{20(M-2)}{N \Delta^{6}}\left(a_{0}+a_{1} \Delta^{2}\right) \\
& \times\left(1+\sqrt{\left\{1+\frac{2 N \Delta^{2}}{5(M-2)}\right.} \frac{1+|\rho|^{2}+2|\rho| \operatorname{Sgn}(\cos \phi)-4|\rho| \operatorname{Sgn}(\cos \phi) \Delta^{2}}{\left.3-|\rho|^{2}+2|\rho| \operatorname{Sgn}(\cos \phi)+\left\{\left[3|\rho|^{2}-66|\rho| \operatorname{Sgn}(\cos \phi)-29\right] / 20\right\} \Delta^{2}\right\}}\right) \\
\simeq & 3\left(a_{0}+a_{1} \Delta^{2}\right) \tilde{\xi}_{T}
\end{aligned}
$$

where $\tilde{\xi}_{T}$ is the resolution threshold for two coherent sources of equal power and same phase given in Reference 12 , and $a_{0}$ and $a_{1}$ are given by

$$
a_{0}=\frac{3-|\rho|^{2}+2|\rho| \operatorname{Sgn}(\cos \phi)}{12}
$$

and

$$
a_{1}=\frac{11-5|\rho|^{2}-2|\rho| \operatorname{Sgn}(\cos \phi)}{48}
$$

with

$$
\operatorname{Sgn}(a)=\left\{\begin{aligned}
1 & \text { for } a \geqslant 0 \\
-1 & \text { for } a<0
\end{aligned}\right.
$$

It can be seen from eqn. 41 that for $|\rho|=1$ and $\phi=0, \zeta$ reduces to $\tilde{\xi}_{\boldsymbol{T}}$.

(b) For $|\sin \phi| \gg \Delta$,

$$
\begin{aligned}
\zeta \simeq & \frac{20(M-2)}{N \Delta^{4}}\left(\frac{3-|\rho|^{2}+2|\rho| \cos \phi}{4 \sin ^{2} \phi}\right) \\
& \times\left(1+\sqrt{\left.\left[1+\frac{2 N \Delta^{2}}{5(M-2)} \frac{1+|\rho|^{2}+2|\rho| \cos \phi}{3-|\rho|^{2}+2|\rho| \cos \phi}\right]\right)}\right. \\
\simeq & \frac{3-|\rho|^{2}+2|\rho| \cos \phi}{4 \sin ^{2} \phi} 3 \Delta^{2} \tilde{\xi}_{T}
\end{aligned}
$$

(c) For $\cos ^{2}\left(\phi-(M-1) \omega_{d}\right) \approx 1$,

$$
\begin{aligned}
\zeta \simeq & \frac{20(M-2)}{N \Delta^{4}}\left(\frac{3-|\rho|^{2}+2|\rho|}{4}\right) \\
& \times \theta^{-2}\left(1+\sqrt{ }\left[1+\frac{2 N \Delta^{2}}{5(M-2)} \frac{1+|\rho|^{2}+2|\rho|}{3-|\rho|^{2}+2|\rho|}\right]\right) \\
\simeq & \frac{3-|\rho|^{2}+2|\rho|}{4} 3 \Delta^{2} \theta^{-2} \tilde{\xi}_{T}
\end{aligned}
$$

where

$$
\theta= \begin{cases}\phi-(M-1) \omega_{d} & \text { for } \phi \simeq(M-1) \omega_{d} \\ (M-1) \omega_{d}-\phi-\pi & \text { for } \phi \simeq(M-1) \omega_{d}-\pi\end{cases}
$$

It can be seen from eqn. 46 that the resolution threshold has a peak value when $\theta$ approaches zero. This result comes from the fact that the MUSIC with forward/ backward smoothing and $K=1$ can not decorrelate the two coherent sources with phase difference equal to $(M-1) \omega_{d}$ or $(M-1) \omega_{d}-\pi$

\section{Forward-only smoothing} of the MUSIC with $K=2$. In the case, $\Gamma_{i k l j}$ in eqn. 13
For forward-only smoothing, we analyse the performance

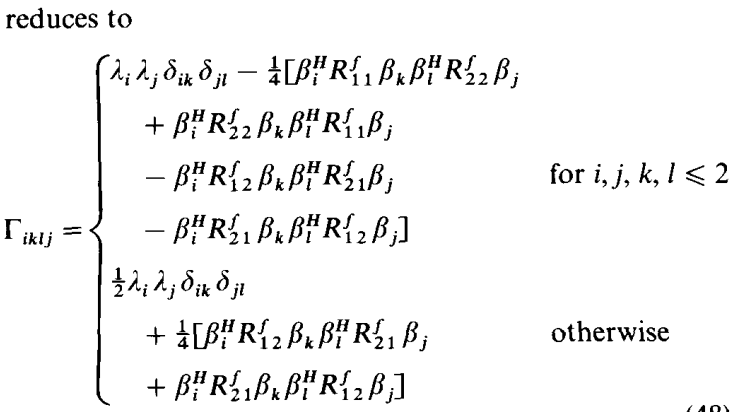

where

$$
\delta_{i j}= \begin{cases}1 & \text { for } i=j \\ 0 & \text { otherwise }\end{cases}
$$

In eqn. $48, \Gamma_{i k l j}$ is not only dependent on the covariance related to the cross correlation matrices $R_{12}^{f}$ and $R_{21}^{f}$. Concerning $R_{21}^{f}$ defind by eqn. 14, it can be written as

$$
R_{21}^{f}=A D R_{u} A^{H}+\sigma^{2} J_{1}
$$

where $J_{1}$ is

$$
J_{1}=\left[\begin{array}{ccccc}
0 & 1 & 0 & \cdots & 0 \\
0 & 0 & 1 & \cdots & 0 \\
\vdots & \vdots & & \ddots & \vdots \\
0 & 0 & \cdots & 0 & 1 \\
0 & \cdots & \cdots & \cdots & 0
\end{array}\right]
$$

As to $R_{12}^{f}$, it is equal to $\left(R_{21}^{f}\right)^{H}$. Substituting eqn. 48 into eqn. 10 and using the following identity (shown in Appendix 9.1),

$$
\sum_{k=3}^{M} \beta_{k}^{H} J_{1} B_{k}=-\sum_{k=1}^{2} \beta_{k}^{H} J_{1} \beta_{k}
$$

it is proved in Appendix 9.2 that the expression for the mean of the null spectrum in the case of forward-only matrices of the two subarrays, $R_{11}^{f}$ and $R_{22}^{f}$, but also 
smoothing and $K=2$ is given by

$$
\begin{aligned}
E[\hat{Q}(\omega)]= & Q(\omega)+\frac{1}{2 N} \sum_{i=1}^{2} \frac{\sigma^{2}}{\left(\lambda_{i}-\sigma^{2}\right)^{2}} \\
& \times\left\{\left[\lambda_{i}(M-2)-\operatorname{Re}\left(\beta_{i}^{H} R_{12}^{f} \beta_{i} \sum_{k=1}^{2} \beta_{k}^{H} J_{1} \beta_{k}\right)\right]\right. \\
& \left.\times\left|\beta_{i}^{H} a(\omega)\right|^{2}-\lambda_{i} Q(\omega)\right\} \\
& +O\left(\frac{1}{N^{2}}\right) \text { for } \omega=\omega_{1}, \omega_{2} \text { and } \omega_{m}
\end{aligned}
$$

Then, using $\left|\beta_{i}^{H} R_{12}^{f} \beta_{i}\right| \leqslant \beta_{i}^{H} \bar{R}_{f} \beta_{i}=\lambda_{i}$ and $\left|\beta_{k}^{H} J_{1} \beta_{k}\right| \leqslant$ $\beta_{k}^{H} \beta_{k}=1$, it can be easily shown that

$$
\left|\operatorname{Re}\left\{\beta_{i}^{H} R_{12}^{f} \beta_{i}\left(\sum_{k=1}^{2} \beta_{k}^{H} J_{1} \beta_{k}\right)\right\}\right| \leqslant 2 \lambda_{i}
$$

Therefore, for large $M$, eqn. 53 may be approximated by

$$
\begin{aligned}
E[\hat{Q}(\omega)] \simeq & Q(\omega)+\frac{1}{2 N} \sum_{i=1}^{2} \frac{\sigma^{2} \lambda_{i}}{\left(\lambda_{i}-\sigma^{2}\right)^{2}} \\
& \times\left[(M-2)\left|\beta_{i}^{H} a(\omega)\right|^{2}-Q(\omega)\right] \\
& +O\left(\frac{1}{N^{2}}\right)
\end{aligned}
$$

Note that eqn. 55 is the same as eqn. 15 which represents the forward/backward smoothing case. Therefore, the expressions for the mean and resolution threshold derived from eqn. 55 have forms similar to eqns. 28,33 and 38 , except that the effective correlation coefficient is different. For forward-only smoothing with $K=2$, the effective correlation coefficient is

$$
\rho_{t}^{f}=e^{j\left(\omega_{d}-\phi\right)} \cos \left(\omega_{d}\right)
$$

Since $\left|\rho_{t}^{f}\right|$ is independent of $\phi$, the bias and resolution threshold of the forward-only smoothing are less sensitive to $\phi$ than those of the forward/backward smoothing. Therefore, using $\Delta \ll 1$, the resolution threshold may be approximated by

$$
\begin{aligned}
\zeta \simeq & \frac{20(M-2)}{N \omega_{d}^{2}} \Delta^{-4}\left(k_{0}+k_{1} \Delta+k_{2} \Delta^{2}\right) \\
& \times\left(1+\sqrt{\left.\left[1+\frac{2 N \Delta^{2}}{5(M-2)} C\right]\right)}\right. \\
\simeq & M^{2}\left(k_{0}+k_{1} \Delta+k_{2} \Delta^{2}\right) \tilde{\xi}_{T}
\end{aligned}
$$

where

$$
\begin{aligned}
& k_{0}= \frac{3-|\rho|^{2}+2|\rho| \cos \phi}{4} \\
& k_{1}= \frac{\sqrt{3}}{2}|\rho| \sin \phi \\
& k_{2}=-\frac{|\rho|^{2}+1+10|\rho| \cos \phi}{16} \\
&+\frac{3-|\rho|^{2}-|\rho| \cos \phi}{4 M^{2}} \\
& C= \frac{1+|\rho|^{2}+2|\rho| \cos \phi}{3-|\rho|^{2}+2|\rho| \cos \phi+2 \sqrt{ }(3)|\rho| \sin \phi \Delta} \\
&+\left\{\left[3|\rho|^{2}-66|\rho| \cos \phi-29\right] / 20\right\} \Delta^{2}
\end{aligned}
$$

It can be seen from eqn. 57 that for $|\rho|=1$ and $\phi=0$, $\zeta \simeq M^{2} \tilde{\xi}_{T}$, where $\tilde{\xi}_{T}$ is the resolution threshold for the forward/backward smoothing with $K=1$ in similar source situations.

\section{Computer simulations}

Computer simulation results are presented in this section. We first simulated the bias and resolution threshold for the forward/backward smoothing with $K=1$. Fig. 1 presents the bias computed from eqn. 28 and the bias averaged from 30 independent simulation trials with $M=10$, $|\rho|=1, \mathrm{SNR}=30 \mathrm{~dB}, N=100$, and $\phi=-60^{\circ}, 0^{\circ}$ and $30^{\circ}$. Fig. 2 shows the bias for $|\rho|=1$ and $|\rho|=0.1$ with $M=10$, SNR of the second source $=20 \mathrm{~dB}, \theta_{1}=69^{\circ}$ and $\theta_{2}=75^{\circ}$. Results show that the computed and averaged biases match very well. The resolution threshold was simulated with parameters the same as those used in Fig. 1 except that $M=7$. Table 1 shows the probability of resolution averaged from a hundred independent trials for different SNR and angular separation. The resolution threshold is chosen to be the SNR where the probability of resolution is 0.5 .

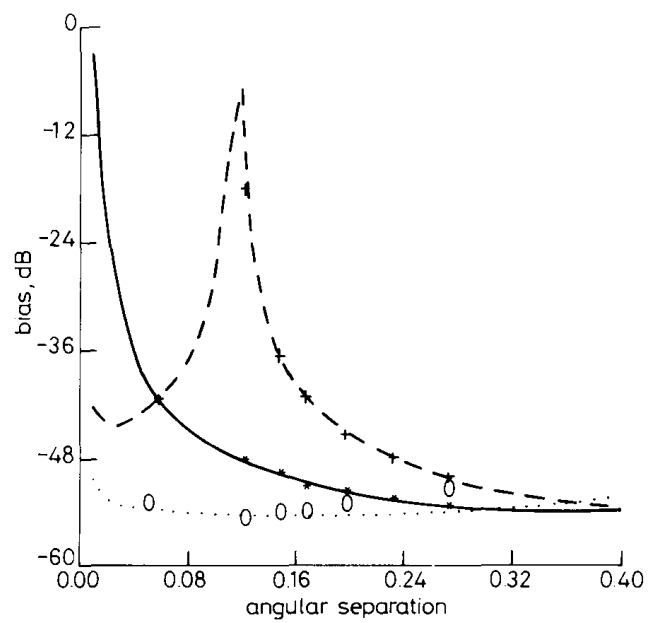

Fig. 1 Bias at one of the arrival angles for forward/backward smoothing

$K=1$, ten sensors, $|\rho|=1$, SNR $=30 \mathrm{~dB}$ and 100 snapshots; $\longrightarrow$ computed bias for $\phi=0^{\circ} ;-\cdots$ computed bias for $\phi=30^{\circ} ; \cdots \cdots$. computed bias for $\phi=-60^{\circ} ;$ averaged bias for $\phi=0^{\circ} ;+$ averaged bias for $\phi=30^{\circ} ; O$ averaged bias for $\phi=-60^{\circ}$

Fig. 3 plots the resolution threshold obtained from Table 1 and that computed from eqn. 38. Fig. 4 shows the resolution threshold simulated with the same parameters as used in Fig. 2. Again, the theoretical results agree with the results obtained from Monte Carlo simulations. From Figs. 3 and 4, it may be observed that the resolution threshold has a peak value for $\phi$ close to $(M-1) \omega_{d}$ or $(M-1) \omega_{d}-\pi$. This result is consistant with the analysis in eqn. 46 . The bias and resolution threshold for the forward-only smoothing with $K=2$ is presented in Figs. 5-8. Here, $M$ is chosen to be ten in simulating both the bias and resolution threshold. Figs. 5 and 6 show the bias simulation results, and Figs. 7 and 8 present the resolution thresholds. From Figs. 4 and 8 , the results show that the resolution threshold of the forward/ backward smoothed case with $K=1$ is lower than that of the forward-only smoothed case with $K=2$ except when $\phi$ is close to $(M-1) \omega_{d}$ or $(M-1) \omega_{d}-\pi$.

IEE PROCEEDINGS-F, Vol. I38, No. 5, OCTOBER I99] 

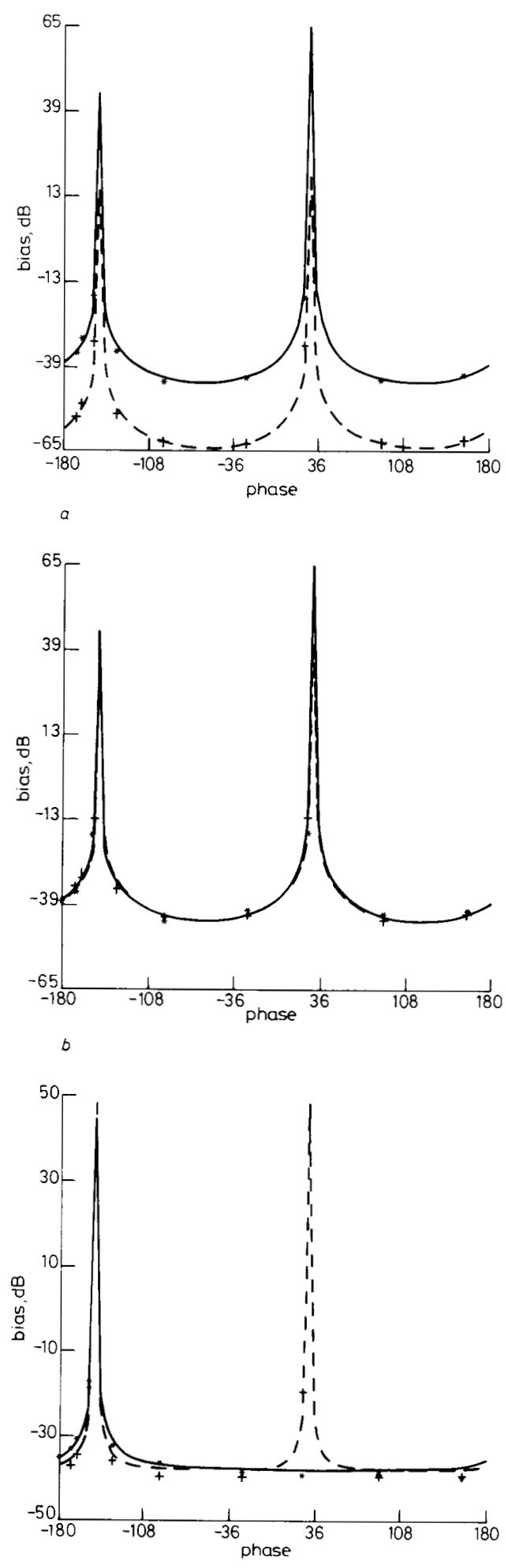

c

Fig. 2 Bias for forward/backward smoothing

$K=1$, ten sensors, 100 snapshots, SNR of the weaker source equal to $20 \mathrm{~dB}$ $\theta_{1}=69^{\circ}$ and $\theta_{2}=75^{\circ} ;-\ldots$ computed bias for $|\rho|=1 ; \ldots-\ldots$ computed bias for $|\rho|=0.1 ; *$ averaged bias for $|\rho|=1 ;+$ averaged bias for $|\rho|=0.1$ $a$ bias at $\omega_{1}$

$c$ bias at $\omega_{2}$

IEE PROCEEDINGS-F, Vol. 138, No. 5, OCTOBER 1991

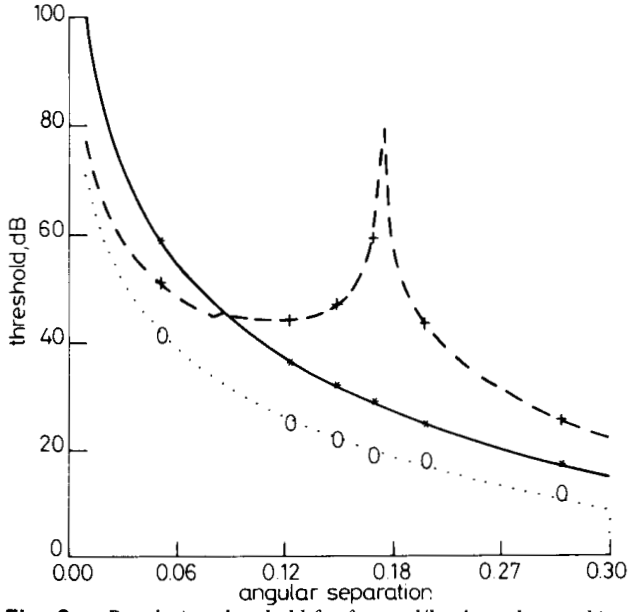

Fig. 3 Resolution threshold for forward/backward smoothing $K=1$, seven sensors, $|\rho|=1$ and 100 snapshots; - - - computed threshold for $\phi=0^{\circ} ;-\cdots-$ computed threshold for $\phi=30^{\circ} ; \cdots \cdots$ computed threshold for $\phi=-60^{\circ} ; \cdot$ averaged threshold for $\phi=0^{\circ} ;+$ averaged threshold for $\phi=30^{\circ}$ $O$ averaged threshold for $\phi=-60^{\circ}$

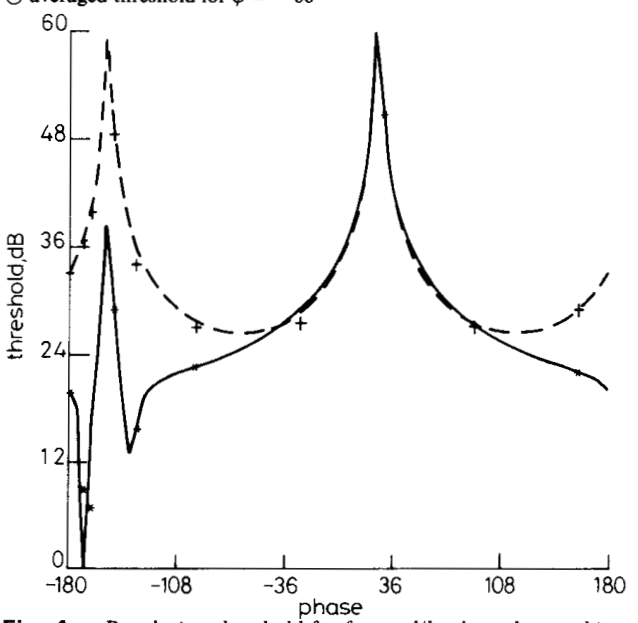

Fig. 4 Resolution threshold for forward/backward smoothing $K=1$, ten sensors, 100 snapshots, $\theta_{1}=69^{\circ}$ and $\theta_{2}=75^{\circ} ;-$ computed thresholds for $|\rho|=1$; $\ldots-$ computed threshold for $|\rho|=0.1$; * averaged threshold for $|\rho|$

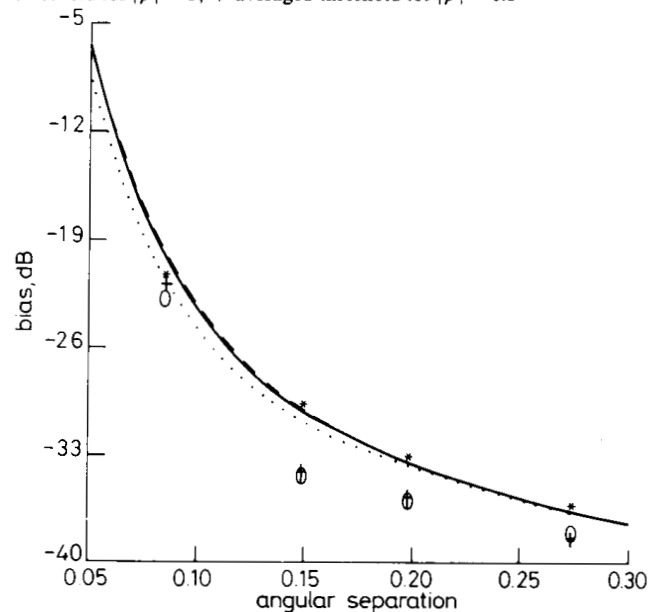

Fig. 5 Bias at one of the arrival angles for forward-only smoothing $K=2$, ten sensors, $|\rho|=1, \mathrm{SNR}=30 \mathrm{~dB}$ and 100 snapshots; - computed bias for $\phi=0^{\circ} ; \ldots-$ computed bias for $\phi=30^{\circ} ; \cdots \cdots$. computed bias for $\phi=-60^{\circ}$; averaged bias for $\phi=0^{\circ} ;+$ averaged bias for $\phi=30^{\circ}$; $O$ averaged bias for $\phi=-60^{\circ}$ 

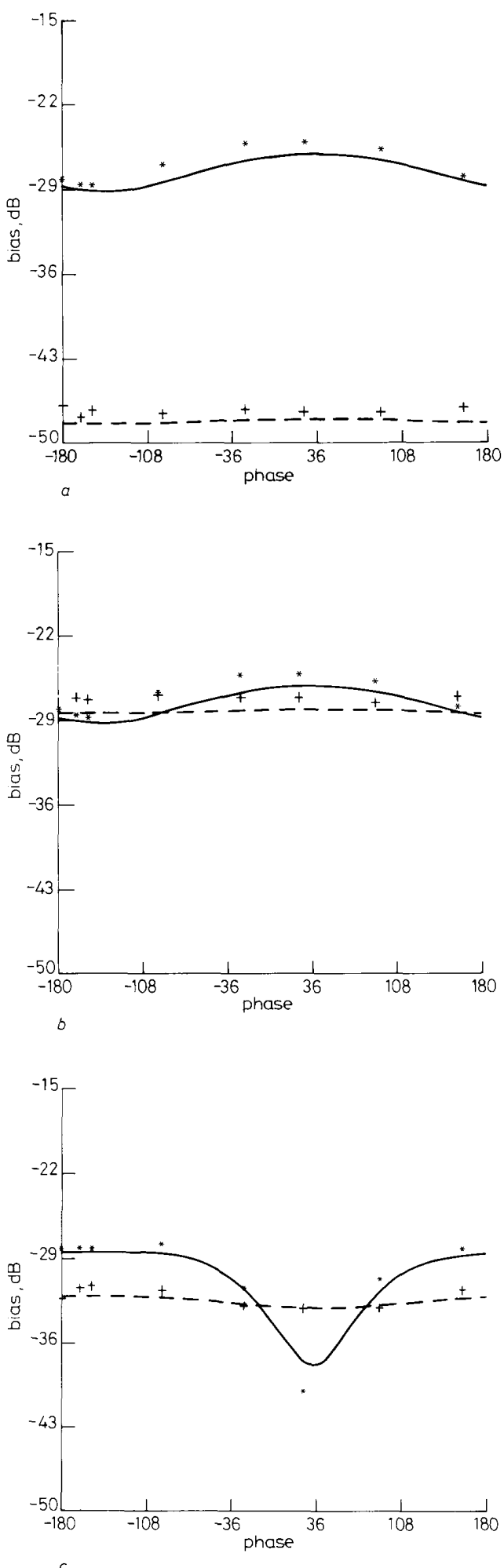

Fig. 6 Bias for forward only smoothing

$K=2$, ten sensors, 100 snapshots, SNR of the weaker source equal to $30 \mathrm{~dB}$ $\theta_{1}=69^{\circ}$ and $\theta_{2}=75^{\circ}$; Computed bias for $|\rho|=1 ;-.--$ computed bias for $|\rho|=0.1 ; *$ averaged bias for $|\rho|=1 ;+$ averaged bias for $|\rho| q 0.1$

a Bias at $\omega_{1}$

$c$ Bias at $\omega_{3}$

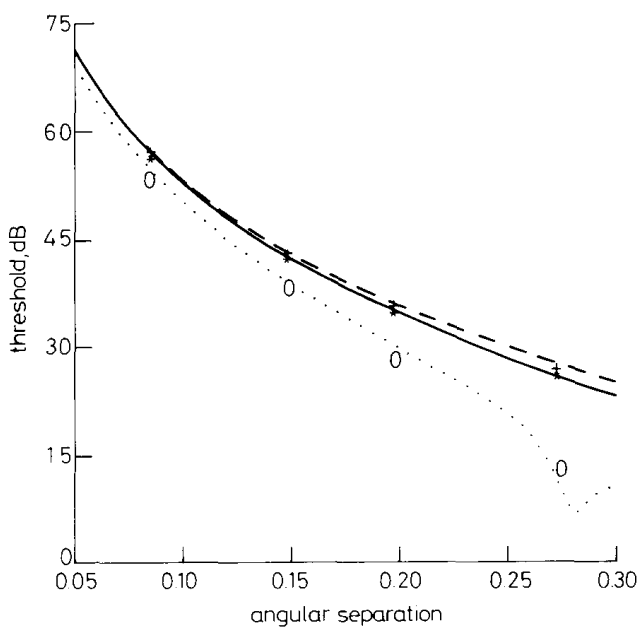

Fig. 7 Resolution threshold for forward only smoothing

$K=2$, ten sensors, $|\rho|=1$ and 100 snapshots; - _ computed threshold for $\phi=0^{\circ}$. . . computed threshold for $\phi=30^{\circ} ; \ldots \ldots$ computed threshold for $\phi=-60^{\circ} ;{ }^{*}$ averaged threshold for $\phi=0^{\circ} ;+$ averaged threshold for $\phi=30^{\circ}$; 0 , averaged threshold for $\phi=-60^{\circ}$

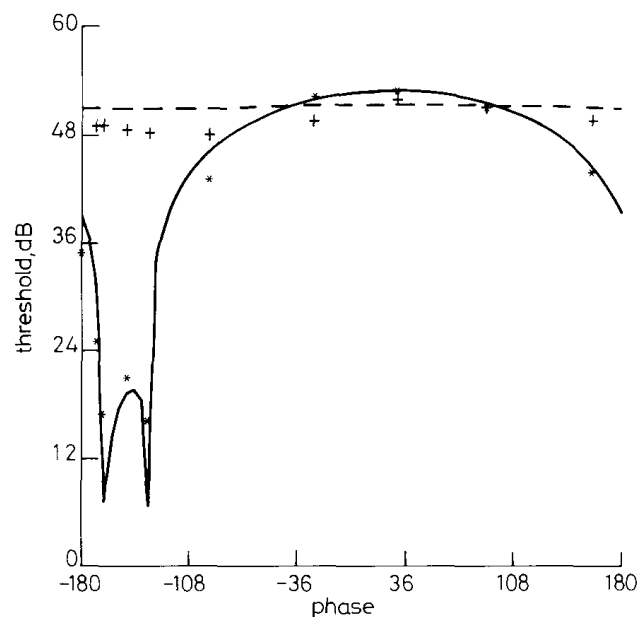

Fig. 8 Resolution threshold for forward only smoothing $K=2$, ten sensors, 100 snapshots, $\theta_{1}=69^{\circ}$ and $\theta_{2}=75^{\circ}$; computed threshold for $|\rho|=1 ;----$ computed threshold for $|\rho|=0.1$; averaged threshold for $|\rho|=1 ;+$ averaged threshold for $|\rho|=0.1$

\section{Conclusion}

We have derived the bias and resolution threshold of the MUSIC method with forward/backward smoothing and forward-only smoothing for two coherent sources with different phase and unequal power. The numbers of subarrays used for derivation are one for forward/backward smoothing and two for forward-only smoothing. Results show that the resolution threshold of the forward/ backward smoothing scheme is highly dependent on the phase difference between the two coherent sources whereas that of the forward-only smoothing is not, except when the power ratio of the two sources is close to 1. The resolution thresholds associated with both of these smoothing schemes also depend on the power ratio of the two sources and are mainly dictated by the SNR of the weaker source. The simulation results indicate that the resolution threshold of the forward/backward smoothing

IEE PROCEEDINGS-F, Vol. 138, No. 5, OCTOBER 199 
Table 1: Resolution probability for forward/backward smoothing with 100 snapshots, 7 sensors, 100 simulations. $\underline{\rho} \mid=1$

\begin{tabular}{|c|c|c|c|c|c|c|c|c|}
\hline \multicolumn{2}{|c|}{$\begin{array}{l}\text { Angles of } \\
\text { arrival }\end{array}$} & \multirow{2}{*}{$\frac{\text { Ang. sep. }}{2 \omega_{d}}$} & \multicolumn{2}{|c|}{$\phi=-60^{\circ}$} & \multicolumn{2}{|c|}{$\phi=0^{\circ}$} & \multicolumn{2}{|c|}{$\phi=30^{\circ}$} \\
\hline$\theta_{1}$ & $\overline{\theta_{2}}$ & & SNR & Prob. & SNR & Prob. & SNR & Prob. \\
\hline 84 & 79 & 0.0514 & $\begin{array}{l}40 \\
41 \\
42 \\
43\end{array}$ & $\begin{array}{l}0.39 \\
0.46 \\
0.57 \\
0.64\end{array}$ & $\begin{array}{l}57 \\
58 \\
59 \\
60\end{array}$ & $\begin{array}{l}0.30 \\
0.37 \\
0.48 \\
0.60\end{array}$ & $\begin{array}{l}49 \\
50 \\
51 \\
52\end{array}$ & $\begin{array}{l}0.28 \\
0.35 \\
0.48 \\
0.62\end{array}$ \\
\hline 71 & 65 & 0.1231 & $\begin{array}{l}23 \\
24 \\
25 \\
26\end{array}$ & $\begin{array}{l}0.34 \\
0.41 \\
0.52 \\
0.59\end{array}$ & $\begin{array}{l}34 \\
35 \\
36 \\
37\end{array}$ & $\begin{array}{l}0.24 \\
0.37 \\
0.53 \\
0.67\end{array}$ & $\begin{array}{l}42 \\
43 \\
44 \\
45\end{array}$ & $\begin{array}{l}0.29 \\
0.39 \\
0.52 \\
0.67\end{array}$ \\
\hline 66 & 60 & 0.1493 & $\begin{array}{l}20 \\
21 \\
22 \\
23\end{array}$ & $\begin{array}{l}0.35 \\
0.40 \\
0.50 \\
0.63\end{array}$ & $\begin{array}{l}29 \\
30 \\
31 \\
32\end{array}$ & $\begin{array}{l}0.22 \\
0.33 \\
0.46 \\
0.59\end{array}$ & $\begin{array}{l}45 \\
46 \\
47 \\
48\end{array}$ & $\begin{array}{l}0.25 \\
0.38 \\
0.56 \\
0.64\end{array}$ \\
\hline 62 & 56 & 0.1694 & $\begin{array}{l}17 \\
18 \\
19 \\
20\end{array}$ & $\begin{array}{l}0.37 \\
0.45 \\
0.52 \\
0.60\end{array}$ & $\begin{array}{l}27 \\
28 \\
29 \\
30\end{array}$ & $\begin{array}{l}0.29 \\
0.41 \\
0.56 \\
0.67\end{array}$ & $\begin{array}{l}57 \\
58 \\
59 \\
60\end{array}$ & $\begin{array}{l}0.28 \\
0.41 \\
0.51 \\
0.55\end{array}$ \\
\hline 56 & 50 & 0.1979 & $\begin{array}{l}15 \\
16 \\
17 \\
18\end{array}$ & $\begin{array}{l}0.33 \\
0.40 \\
0.46 \\
0.59\end{array}$ & $\begin{array}{l}23 \\
24 \\
25 \\
26\end{array}$ & $\begin{array}{l}0.25 \\
0.38 \\
0.52 \\
0.68\end{array}$ & $\begin{array}{l}41 \\
42 \\
43 \\
44\end{array}$ & $\begin{array}{l}0.16 \\
0.26 \\
0.47 \\
0.65\end{array}$ \\
\hline 48 & 41 & 0.2736 & $\begin{array}{r}9 \\
10 \\
11 \\
12\end{array}$ & $\begin{array}{l}0.38 \\
0.41 \\
0.49 \\
0.56\end{array}$ & $\begin{array}{l}15 \\
16 \\
17 \\
18\end{array}$ & $\begin{array}{l}0.28 \\
0.40 \\
0.48 \\
0.60\end{array}$ & $\begin{array}{l}24 \\
25 \\
26 \\
27\end{array}$ & $\begin{array}{l}0.26 \\
0.45 \\
0.60 \\
0.69\end{array}$ \\
\hline
\end{tabular}

with $K=1$ is usually smaller than that of the forwardonly smoothing with $K=2$ except when the absolute value of the correlation coefficient is very close to unity, i.e. the phase difference between the two coherent sources is close to $(M-1) \omega_{d}$ or $(M-1) \omega_{d}-\pi$.

\section{Acknowledgment}

The authors wish to thank professor Pillai for his valuable discussions.

\section{References}

1 PISARENKO, V.F.: 'The retrieval of harmonics from a covariance function', Geophy. J.R. Astr. Soc., 1973, 33, pp. 347-366

2 REDDI, S.S. : 'Multiple source location - a digital approach', IEEE Trans., 1979, AES-15, (1), pp. 95-105

3 SCHMIDT, R.O.: 'Multiple emitter location and signal parameter estimation', IEEE Trans., 1986, AP-34, (3), pp. 276-280

4 KUMARESAN, R., and TUFTS, D.W.: 'Estimation the angles of arrival of multiple plane waves', IEEE Trans., 1983, AES-19, (1), pp. $134-139$

5 PAUlRAJ, A., ROY, R., and KAILATH, T.: 'A subspace rotation approach to signal parameter estimation', Proc. IEEE, 1986, 74, pp. $1340-1342$

6 ROY, R., and KAILATH, T.: 'ESPRIT - Estimation of signal parameters via rotation invariance techniques', IEEE Trans., 1989 ASSP-37, (7), pp. 984-995

7 SHAN, T.J., WAX, M., and KAILATH, T.: 'On spatial smoothing for directions-of-arrival estimation of coherent signals', IEEE Trans., 1985, ASSP-33, (8), pp. 806-811

8 PILLAI, S.U., and KWON, B.H.: 'Forward/backward spatial smoothing techniques for coherent signal identification', IEEE Trans., 1989, ASSP-37, (1), pp. 8-15

9 HABER, F., and ZOLTOWSKI, M.: 'Spatial spectrum estimation in a coherent signal environment using an array in motion', IEEE Trans. Antennas \& Prop., Special Issue on adaptive antenna processing system, 1986, AP-34, (3), pp. 301-310

IEE PROCEEDINGS-F, Vol. 138, No. 5, OCTOBER 1991
$10 \mathrm{KAVEH}, \mathrm{M}$., and BARABELL, A.J.: 'The statistical performance of the MUSIC and the minimum-norm algorithms in resolving plane waves in noise', IEEE Trans., 1986, ASSP-34, (4), pp. 331-341

11 PILLAI, S.U.: 'Array signal processing' (Springer-Verlag, 1989)

12 PILLAI, S.U., and KWON, B.H.: 'Performance analysis of MUSICtype high resolution estimations for direction finding in correlated and coherent scenes', IEEE Trans., 1989, ASSP-37, (8), pp. 11761189

13 LEE, H.B., and WENGROVITZ, M.S.: 'Resolution threshold of beamspace MUSIC for two closely spaced emitters', IEEE Trans., 1990, ASSP-38, (9), pp. 1545-1559

\section{$9 \quad$ Appendix}

9.1 Derivation of eqn. 52

Since $\beta_{k}, k=1, \ldots, M$, are a set of orthogonal vectors,

$$
\left[\begin{array}{llll}
\beta_{1} & \beta_{2} & \cdots & \beta_{M}
\end{array}\right]\left[\begin{array}{c}
\beta_{1}^{H} \\
\beta_{2}^{H} \\
\vdots \\
\beta_{M}^{H}
\end{array}\right]=I
$$

Define

$$
d_{i}=\left[\begin{array}{ll}
\beta_{i 1} & \beta_{i 2}
\end{array}\right]^{T}
$$

and

$$
r_{i}=\left[\begin{array}{lll}
\beta_{i 3} & \cdots & \beta_{i M}
\end{array}\right]^{T}
$$

where $\beta_{i k}, i, k=1,2, \ldots, M$, is the $i$ th element of the vector $\beta_{k}, k=1,2, \ldots, M$. Using eqns. 63 and 64 , eqn. 62 can be written as

$$
\left[\begin{array}{cc}
d_{1}^{H} & r_{1}^{H} \\
d_{2}^{H} & r_{2}^{H} \\
\vdots & \vdots \\
d_{M}^{H} & r_{M}^{H}
\end{array}\right]\left[\begin{array}{llll}
d_{1} & d_{2} & \cdots & d_{M} \\
r_{1} & r_{2} & \cdots & r_{M}
\end{array}\right]=I
$$

In eqn. 65 , the elements off the main diagonal are zeros. Therefore, we have

$$
d_{i}^{H} d_{i+1}+r_{i}^{H} r_{i+1}=0
$$

for $i=1,2, \ldots, M-1$. Using eqn. 64 , the right-hand side of eqn. 52 becomes

$$
\begin{aligned}
\sum_{k=3}^{M} \beta_{k}^{H} J_{1} \beta_{k} & =\sum_{k=3}^{M}\left(\sum_{i=1}^{M-1} \beta_{i k}^{*} \beta_{(i+1) k}\right) \\
& =\sum_{i=1}^{M-1}\left(\sum_{k=3}^{M} \beta_{i k}^{*} \beta_{(i+1) k}\right) \\
& =\sum_{i=1}^{M-1} r_{i}^{H} r_{i+1}
\end{aligned}
$$

Using eqn. 66 , eqn. 67 can be written as

$$
\begin{aligned}
\sum_{k=3}^{M} \beta_{k}^{H} J_{1} \beta_{k} & =-\sum_{i=1}^{M-1} d_{i}^{H} d_{i+1} \\
& =-\sum_{k=1}^{2}\left(\sum_{i=1}^{M-1} \beta_{i k}^{*} \beta_{(i+1) k}\right) \\
& =-\sum_{k=1}^{2} \beta_{k}^{H} J_{1} \beta_{k}
\end{aligned}
$$

which is eqn. 52 . 
9.2 Derivation of eqn. 53

Substituting eqn. 48 into eqn. 10 we get

$$
\begin{aligned}
E[\hat{Q}(\omega)]= & Q(\omega)+\frac{1}{N} \sum_{i=1}^{2}\left[\sum_{\substack{k=1 \\
k \neq i}}^{M} \frac{\Gamma_{i i k k}^{f}}{\left(\lambda_{i}-\lambda_{k}\right)^{2}}\left|\beta_{i}^{H} a(\omega)\right|^{2}\right. \\
& \left.-\sum_{\substack{k=1 \\
k \neq i}}^{M} \sum_{\substack{l=1 \\
l \neq i}}^{M} \frac{\Gamma_{i i k l}^{f}}{\left(\lambda_{i}-\lambda_{k}\right)\left(\lambda_{i}-\lambda_{l}\right)} a^{H}(\omega) \beta_{k} \beta_{l}^{H} a(\omega)\right] \\
& +O\left(\frac{1}{N^{2}}\right) \\
= & Q(\omega)+\frac{1}{N} \\
& \times \sum_{i=1}^{2}\left\{A_{i}+B_{i}+C_{i}+D_{i}+E_{i}+F_{i}+G_{i}\right\} \\
& +O\left(\frac{1}{N^{2}}\right)
\end{aligned}
$$

Here,

$$
\begin{aligned}
A_{i}= & \sum_{k=3}^{M} \frac{1}{2} \frac{\lambda_{i} \sigma^{2}}{\left(\lambda_{i}-\sigma^{2}\right)^{2}}\left(\left|\beta_{i}^{H} a(\omega)\right|^{2}-\left|\beta_{k}^{H} a(\omega)\right|^{2}\right) \\
= & \frac{1}{2} \frac{\lambda_{i} \sigma^{2}}{\left(\lambda_{i}-\sigma^{2}\right)^{2}}\left\{(M-2)\left|\beta_{i}^{H} a(\omega)\right|^{2}-Q(\omega)\right\} \\
B_{i}= & \frac{1}{4} \sum_{k=3}^{M} \frac{\beta_{i}^{H} R_{12}^{f} \beta_{i} \beta_{k}^{H} R_{21}^{f} \beta_{k}+\beta_{i}^{H} R_{21}^{f} \beta_{i} \beta_{k}^{H} R_{12}^{f} \beta_{k}}{\left(\lambda_{i}-\sigma^{2}\right)^{2}} \\
& \times\left|\beta_{i}^{H} a(\omega)\right|^{2} \\
= & -\operatorname{Re}\left\{\frac{\sigma^{2}\left(\beta_{i}^{H} R_{12}^{f} \beta_{i}\right)}{2\left(\lambda_{i}-\sigma^{2}\right)^{2}}\right. \\
& \left.\times\left(\sum_{k=1}^{2} \beta_{k}^{H} J_{1} \beta_{k}\right)\left|\beta_{i}^{H} a(\omega)\right|^{2}\right\}
\end{aligned}
$$

where $J_{1}$ is defined by eqn. 51 , and

$$
C_{i}=\sum_{\substack{k=1 \\ k \neq i}}^{2} \frac{\lambda_{i} \lambda_{k}}{\left(\lambda_{i}-\lambda_{k}\right)^{2}}\left(\left|\beta_{i}^{H} a(\omega)\right|^{2}-\left|\beta_{k}^{H} a(\omega)\right|^{2}\right)
$$

$$
\begin{aligned}
D_{i}= & \frac{-1}{4} \sum_{k=3}^{M} \sum_{\substack{l=1 \\
l \neq i}}^{M} \frac{\beta_{i}^{H} R_{12}^{f} \beta_{i} \beta_{k}^{H} R_{21}^{f} \beta_{l}+\beta_{i}^{H} R_{21}^{f} \beta_{i} \beta_{k}^{H} R_{12}^{f} \beta_{l}}{\left(\lambda_{i}-\sigma^{2}\right)\left(\lambda_{i}-\lambda_{l}\right)} \\
& \times a^{H}(\omega) \beta_{k} \beta_{l}^{H} a(\omega) \\
E_{i}= & \frac{-1}{4} \sum_{\substack{k=1 \\
k \neq i}}^{2} \frac{\left|\beta_{i}^{H} a(\omega)\right|^{2}}{\left(\lambda_{i}-\lambda_{k}\right)^{2}}\left\{\beta_{i}^{H} R_{11}^{f} \beta_{i} \beta_{k}^{H} R_{22}^{f} \beta_{k}\right. \\
& +\beta_{i}^{H} R_{22}^{f} \beta_{i} \beta_{k}^{H} R_{11}^{f} \beta_{k}-\beta_{i}^{H} R_{12}^{f} \beta_{i} \beta_{k}^{H} R_{21}^{f} \beta_{k} \\
& \left.-\beta_{i}^{H} R_{21}^{f} \beta_{i} \beta_{k}^{H} R_{12}^{f} \beta_{k}\right\} \\
F_{i}= & \frac{1}{4} \sum_{\substack{k=1 \\
k \neq i}}^{2} \sum_{\substack{l=1 \\
l \neq i}}^{2} \frac{a^{H}(\omega) \beta_{k} \beta_{l}^{H} a(\omega)}{\left(\lambda_{i}-\lambda_{k}\right)\left(\lambda_{i}-\lambda_{l}\right)} \\
& \times\left\{\beta_{i}^{H} R_{11}^{f} \beta_{i} \beta_{k}^{H} R_{22}^{f} \beta_{1}+\beta_{i}^{H} R_{22}^{f} \beta_{i} \beta_{k}^{H} R_{11}^{f} \beta_{l}\right. \\
& \left.-\beta_{i}^{H} R_{12}^{f} \beta_{i} \beta_{k}^{H} R_{21}^{f} \beta_{1}-\beta_{i}^{H} R_{21}^{f} \beta_{i} \beta_{k}^{H} R_{12}^{f} \beta_{l}\right\} \\
G_{i}= & \frac{-1}{4} \\
& \times \sum_{\substack{k=1 \\
k \neq i}}^{2} \sum_{\substack{l=3 \\
\text { ind }}}^{M} \frac{\beta_{i}^{H} R_{12}^{f} \beta_{i} \beta_{k}^{H} R_{21}^{f} \beta_{l}+\beta_{i}^{H} R_{21}^{f} \beta_{i} \beta_{k}^{H} R_{12}^{f} \beta_{l}}{\left(\lambda_{i}-\lambda_{k}\right)\left(\lambda_{i}-\lambda_{l}\right)} \\
& \times a^{H}(\omega) \beta_{k} \beta_{l}^{H} a(\omega)
\end{aligned}
$$

Since $\sum C_{i}=0, \sum\left(E_{i}+F_{i}\right)=0$, and $D_{i}$ and $G_{i}$ are close to zero for $\omega$ equal to $\omega_{1}, \omega_{2}$ and $\omega_{m}$, we have from eqn. 69

$$
\begin{aligned}
E[\hat{Q}(\omega)]= & Q(\omega)+\frac{1}{2 N} \sum_{i=1}^{2} \frac{\sigma^{2}}{\left(\lambda_{i}-\sigma^{2}\right)^{2}} \\
& \times\left\{\left[\lambda_{i}(M-2)-\operatorname{Re}\left(\beta_{i}^{H} R_{12}^{f} \beta_{i} \sum_{k=1}^{2} \beta_{k}^{H} J_{1} \beta_{k}\right)\right]\right. \\
& \left.\times\left|\beta_{i}^{H} a(\omega)\right|^{2}-\lambda_{i} Q(\omega)\right\} \\
& +O\left(\frac{1}{N^{2}}\right) \text { for } \omega=\omega_{1}, \omega_{2} \text { and } \omega_{m}
\end{aligned}
$$

